\title{
DESCRIPTION OF ORIENTATION DISTRIBUTIONS OF CUBIC CRYSTALS BY MEANS OF 3-D ROTATION COORDINATES
}

\author{
GERHARD IBE and KURT LÜCKE \\ Leichtmetall-Forschungsinstitut of the Vereinigte Aluminium-Werke AG, Bonn, Germany, and \\ Institut für Allgemeine Metallkunde und Metallphysik, Technische Hochschule, Aachen, Germany
}

(Received January 13, 1972, in final form March 29, 1972)

\begin{abstract}
A method has been described to represent orientations and orientation distributions of cubic crystals by means of a 3-dimensional orientation space which is formed by the repeatedly discussed rotation coordinates (axis and angle of rotation). Special emphasis has been given to the problem of multi-valency of the representation due to the cubic symmetry, to the description of scattering around an ideal orientation and to the numerical evaluation of these orientation coordinates by means of rotation matrices.
\end{abstract}

\section{INTRODUCTION}

For the description of the orientation of a crystal, one needs 3 parameters related to a reference coordinate system. The reference system is mostly chosen either with respect to the geometry of the specimen (e.g. for rolled sheets the system rolling direction, sheet normal and cross direction), or with respect to the crystallographic orientation of another crystal (e.g. for a recrystallized grain the crystallographic axes of the deformed matrix crystal).

The 3 parameters can be chosen in many different ways. One can, for example, imagine that the reference coordinate system is transformed into the system of the crystallographic axes of the considered crystal by rotating it around a certain axis and uses the polar coordinates $\varphi$ and $\vartheta$ of the axis of rotation and the angle of rotation $\psi$. Another possibility is to apply the 3 Eulerian angles, which are obtained if the reference system is turned into the orientation of the crystal by 3 sequential rotations around its orthogonal axes. As a third example, in the case of rolled sheets, one often uses the indices $\{h, k, l\}\langle u, v, w\rangle$ which represent the lattice plane of the crystal coinciding with the plane of rolling (2 parameters) and the crystallographic direction falling into the direction of rolling (1 parameter).

Such a set of 3 orientation parameters can be considered as a coordinate system defining a threedimensional orientation space. An orientation can then be treated as a point and, correspondingly, an orientation distribution as a density distribution in such an orientation space. This 3-dimensional method of description of orientation distributions has been used by Lücke, Perlwitz and Pitsch, ${ }^{1}$ and in a similar manner by Williams. ${ }^{2}$ They have applied the rolling parameters $\{h, k, l\}\langle u, v, w\rangle$ and their distribution to describe rolling textures. Viglin $^{3}$ and Bunge et al., ${ }^{4-8}$ also Roe and others $^{9-14}$ have discussed orientation representations with the aid of the Eulerian angles.

Mostly, orientation distributions, e.g. those of crystallites of polycrystalline metals (textures), are described by pole figures. These are stereographic projections of the density distribution of the normals (poles) to certain crystallographic planes $\{h, k, l\}$ over the various directions within the sample. The reason for the preference of the pole figures is that these can be determined in rather simple ways by X-ray methods. Since, however, a pole figure is only a kind of 2-dimensional projection of the 3-dimensional orientation variety it does not contain the full information on the orientation distribution. If, for instance, a certain point in the pole figure representing a pole $\{h, k, l\}$ of a crystal is considered, it is not known which other points of the pole figure belong to the same crystal, i.e. nothing is known about the rotation of the crystal around this axis. Only in cases of very pronounced textures with only few components can one guess from the pole figure the approximate orientation of the crystals. In more complicated cases, one has to apply a 3-parameter analysis.

It is the subject of this paper to discuss the method of such a 3-parameter analysis of orientation dis- 
tributions using the above-mentioned 3-dimensional rotation coordinates $\varphi, \vartheta$ and $\psi$. Compared to the Eulerian coordinates which represent a sequence of 3 rotations, these coordinates describe a single rotation which is all necessary for the same purpose. Even though these coordinates had frequently been discussed previously ${ }^{15-18}$ (mainly by Mackenzie), the method of using them for the practical analysis of orientation distributions was not sufficiently developed.

Therefore, in the present paper a systematic derivation of the general theoretical relationships needed for such an analysis will be given. In a second paper $^{19}$ (in the following referred to as Part II) an example (recrystallization of deformed $\mathrm{Fe}-3 \% \mathrm{Si}$ single crystals) will be treated in order to demonstrate how the orientation parameters $\varphi, \vartheta, \psi$ can be determined numerically and be used for a rational description of orientation distributions. Finally, at the end of Part II, a general discussion of the principles of describing orientation distributions will be given.

\section{DESCRIPTION OF ORIENTATIONS IN CUBIC CRYSTALS BY MEANS OF ROTATIONAL COORDINATES}

For any given orientation of a crystal $B$ with respect to a reference orientation $A$, the coordinates $\varphi, \vartheta$ and $\psi$ of the corresponding 3-dimensional rotation $R(\varphi, \vartheta, \psi)$ can assume the following values:

$$
0 \leqq \varphi \leqq 2 \pi ; \quad 0 \leqq \vartheta \leqq \pi / 2 ; \quad-\pi \leqq \psi \leqq+\pi
$$

(positive sign of $\psi$ is to indicate clockwise rotation). The corresponding orientation space can, for instance, be represented in such a way that the unit vector $\mathbf{v}$ of the rotation axis (with the coordinates $\varphi$ and $\vartheta$ ) is plotted in the usual stereographic projection and the rotation angle $\psi$, as a third coordinate, perpendicular to the projection plane (Figure 1a).

Another representation of the orientation space is obtained if -in the manner of 3-dimensional polar coordinates-the angle of rotation $\psi$ is plotted as radius vector in direction of the rotation axes (Figure 1b). By assigning to the counter direction the reversed sense of rotation one obtains instead of the cylindrical orientation space of Figure 1a a sphere with the radius $\psi_{\max }=\pi$ and the somewhat modified range of values $0 \leqq \varphi \leqq 2 \pi$, $0 \leqq \vartheta \leqq \pi, 0 \leqq \psi \leqq \pi$. While the representation
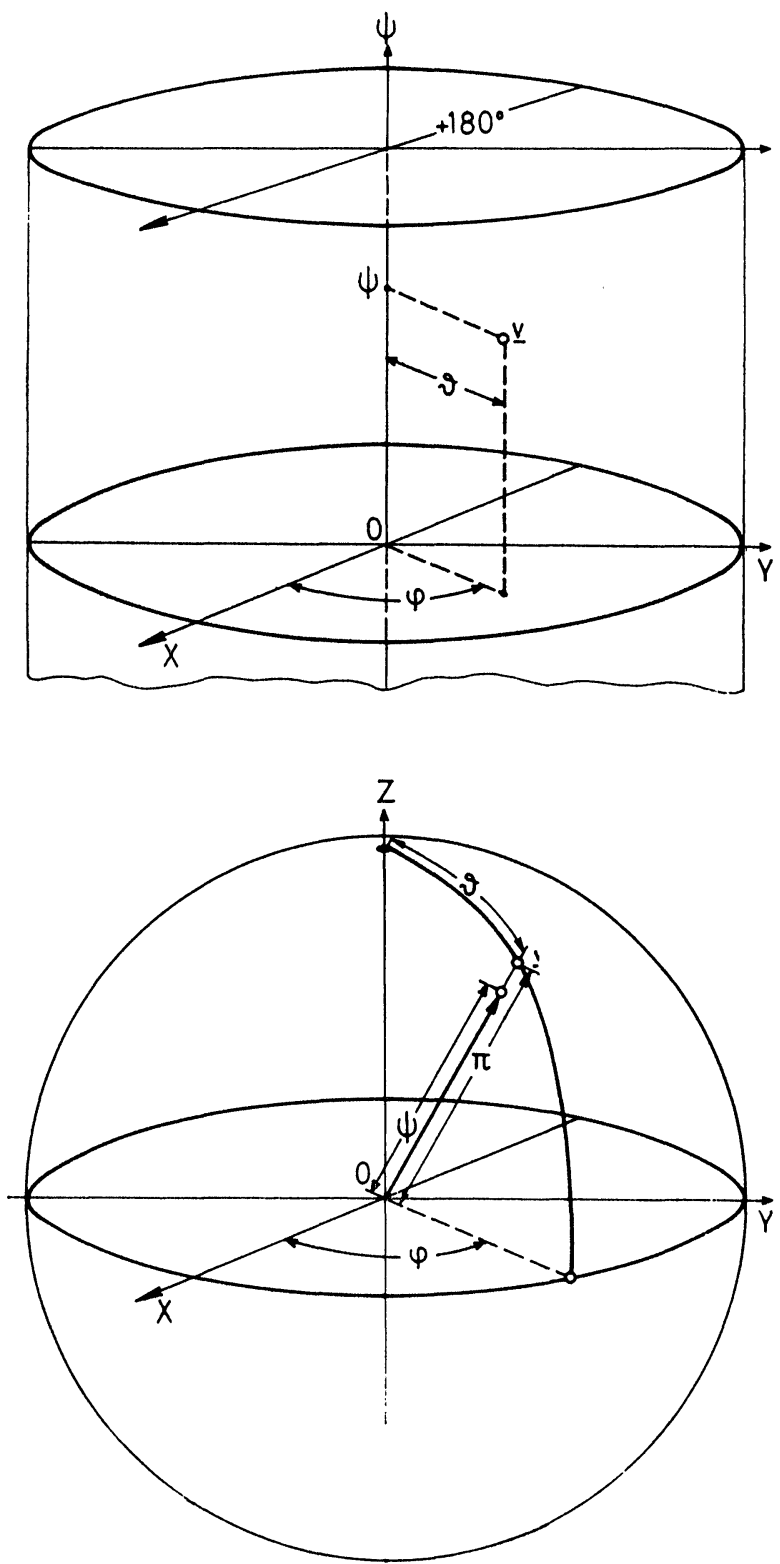

FIGURE 1 Representations of the three-dimensional orientation space formed by the rotational coordinates $(\mathrm{v}, \psi)=(\varphi, \vartheta, \psi)$. (a) cylindrical space, (b) spherical space.

of Figure 1a will prove itself suitable for the practical evaluation of experiments (see Part II) the representation of Figure $1 \mathrm{~b}$ will be advantageous for theoretical considerations. As will be discussed later, in some cases modified rotation angles

$$
\psi^{*}=\left[\frac{18}{\pi^{2}} \cdot(\psi-\sin \psi)\right]^{1 / 3} ; \quad \psi^{* *}=\operatorname{tg}\left(\frac{\psi}{4}\right)
$$


will be used leading to a radius of the orientation sphere of $\psi_{\max }^{*}=(18 / \pi)^{1 / 3}$ and $\psi_{\max }^{* *}=1$.

Since the 3 cube axes of a cubic crystal are nondistinguishable, one obtains 24 different rotations $\left(R_{1}\right.$ to $\left.R_{24}\right)$ for describing one and the same orientation. This corresponds to 24 different possibilities of transforming cube poles of one crystal into those of the other. (These can easily be seen: a first cubic half axis of crystal $A$ can be rotated into six different half axes of crystal $B$ and for each of these rotations a second half axis of $A$ into four different half axes of $B$ ). However, since already one of these 24 rotations suffices to define the orientation, the problem arises which of the 24 rotations, i.e. which of the (theoretically equally well suited) 24 sets of values $\varphi, \vartheta$ and $\psi$ is the most practical one to use. This is different for different cases and will be discussed in Part II.

Mostly in literature, that of the 24 rotations which is distinguished by the smallest angle of rotation $\left(\psi_{\min }\right)$ has implicitly been used; it is named "disorientation" ${ }^{5} R_{\min }$. In the orientation space, Figure 1a, the orientations $R_{\min }$ are located near the plane $\psi=0$ and form there a sub-space in which all possible orientations are contained. $\dagger$ The boundaries of this sub-space, which are curved areas lying symmetrically with respect to the plane $\psi=0$, represent the largest values the angle $\psi_{\min }$ can assume for the various directions of the rotation axis. These angles $\psi_{\min }$ which are indicated in Figure 4 for the unit triangle range between $45^{\circ}$ and $62.8^{\circ}$. In a corresponding way also, for the spherical orientation space Figure $1 \mathrm{~b}$ a sub-space (around the center) containing all the rotations $R_{\text {min }}$ can be defined.

The axes of rotation can be found graphically. For each of the 3 pairs of cube poles which are transformed into each other by the considered rotation, the largest circle which runs symmetrically between the two poles is drawn. The common intersection of the 3 circles gives the corresponding axis of rotation. One of the 24 possibilities is shown in Figure 2 by the circles $G_{1}, G_{2}, G_{3}$ intersecting in v. The rotation angle $\psi$ can then easily be determined if the whole pole figure is rotated in such a way that $\mathbf{v}$ is located in its center.

Less time-consuming is a numerical way to determine the rotational coordinates from the 3 angles $\omega_{1}, \omega_{2}$ and $\omega_{3}$ between the cube poles of $A$ and $B$ to be transformed into each other (compare

$\dagger$ There are many different possibilities to divide the orientation space into 24 sub-spaces in such a way that each sub-space contains each possible orientation once. ${ }^{20}$

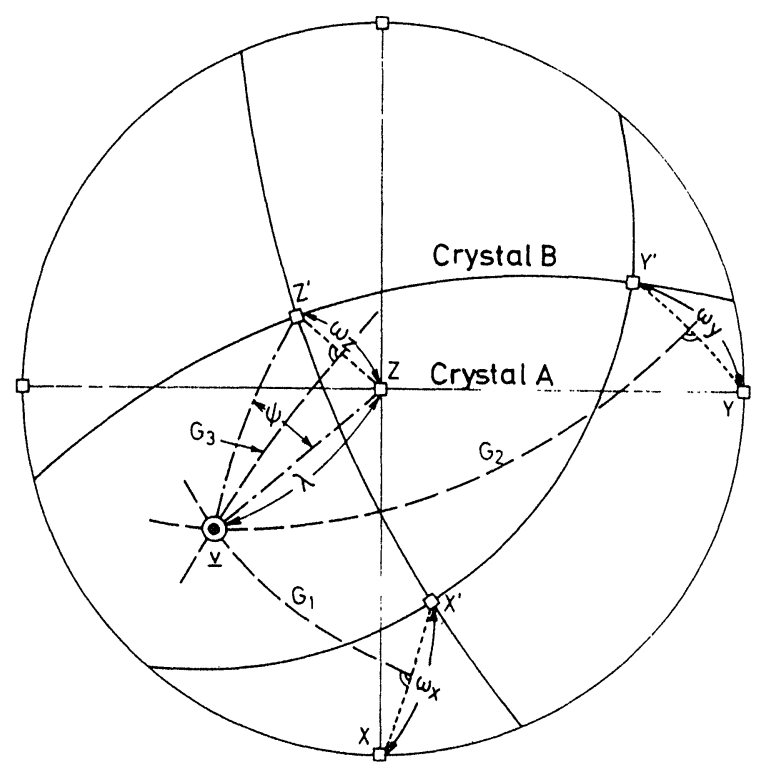

FIGURE 2 Graphic determination of axis ( $v$ ) and angle $(\psi)$ of rotation for transforming the initial orientation $A$ (standard projection with the cube poles $X, Y, Z$ ) into the final orientation $B$ (with the cube poles $X^{\prime}, Y^{\prime}, Z^{\prime}$ ). Relation between rotation angle $\psi$, distance $\lambda$ from pole to rotation axis and distance $\omega$ of the two poles to be transformed into each other (here $Z$ and $Z^{\prime}$ ).

also Figure 5). For a given rotation one obtains here the 3 equations

$$
\sin \left(\frac{\psi}{2}\right)=\frac{\sin \left(\omega_{i} / 2\right)}{\sin \lambda_{i}}
$$

( $i=1,2,3)$ where $\lambda_{i}$ is the angle between the considered pole and the rotation axis. This can be recognized by considering the spherical triangle in in Figure 2 where $\omega_{i}=\omega_{z}$. From Eq. (2), it follows

and, since

$$
\cos ^{2} \lambda_{i}=1-\frac{\sin ^{2}\left(\omega_{i} / 2\right)}{\sin ^{2}(\psi / 2)}
$$

$$
\begin{array}{r}
\sum_{i} \cos ^{2} \lambda_{i}=1, \text { one obtains for the angle } \psi \\
\cos \omega_{1}+\cos \omega_{2}+\cos \omega_{3}=1+2 \cos \psi
\end{array}
$$

From Eqs. (2) and (3) also the Cartesian coordinates $v_{i}=\cos \lambda_{i}$ of the axis of rotation can be obtained.

Figure 3 gives an example showing all 24 axes and angles of rotation which turn orientation $A$ into $B$. One recognizes that in each of the 24 unit triangles of the pole figure just one axis is situated. One further recognizes that all axes $\mathbf{v}$ are situated in groups on largest circles, which run symmetrically between the crystallographic poles to be transformed 
into each other. (Two such circles are shown as dotted lines in Figure 3.) The number of axes on one circle is equal to the crystallographic frequency of the considered type of pole if the axis $v_{\min }$ corresponding to the smallest angle of rotation $\left(\psi_{\min }\right)$ is counted for all of these groups. One has always a total of 13 such largest circles, namely, there are as many as four-, three- and two-fold axes together.

For describing an identical orientation, one can apply the rotation angle $\psi=0$, but also the rotations $\psi=90^{\circ}, 180^{\circ}$ and $270^{\circ}$ around the axes $\langle 100\rangle, 120^{\circ}$ and $240^{\circ}$ around $\langle 111\rangle$ and $180^{\circ}$ around $\langle 110\rangle$. By considering the crystallographic frequency $z$ of the axes $(3$ for $\langle 100\rangle, 4$ for $\langle 111\rangle$ and 6 for $\langle 110\rangle$, one obtains 24 rotations, which represent the actual cubic symmetry operations. For $\psi=0$ a degeneration occurs insofar as then the location of the rotation axis is no longer defined, but any direction may be selected as rotation axis. This degeneration shows up already at finite but small values of $\psi$ : in Eq. (2) a small change in orientation $d \sin (\omega / 2)$ corresponds to a large displacement $d \sin \lambda$ of the rotation axis if $\psi$ is small. In the spherical orientation space (Figure $1 \mathrm{~b}$ ) the orientations with $\psi=0$ correspond to the

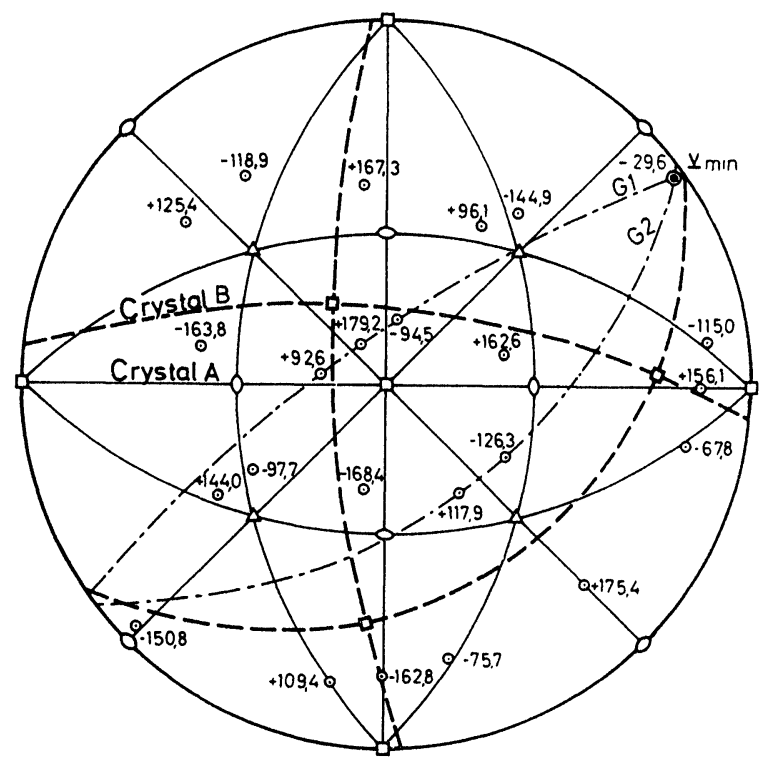

FIGURE 3 Example showing the 24 rotations $R_{i}$ which describe an orientation $B$ with respect to the standard orientation $A$. At the rotation axes (1 to 24 ) the respective rotation angles are indicated; $\mathbf{v}_{\min }$ is emphasized. Of the 13 largest circles connecting $\mathbf{v}_{\min }$ with the various groups of rotation axes, two are plotted as examples (dotted lines $G_{1}$ and $G_{2}$ ). origin of the coordinate system for which, of course, no direction of the rotation axis is defined.

\section{DESCRIPTION OF ORIENTATION DISTRIBUTIONS}

Now let us consider an orientation distribution. According to Dunn ${ }^{21}$ one can define the pole density on the surface of a unit sphere by

$$
D(P)=\frac{d H(P)}{d \Omega}
$$

Here, $d H(P)$ is proportional to the volume fraction of the specimen with poles of the considered type in the space angle element $d \Omega=\sin \vartheta d \vartheta d \varphi$. The normalization is chosen in such a way that

$$
\int_{\varphi=0}^{2 \pi} \int_{\vartheta=0}^{\pi / 2} D(P) \cdot \sin \vartheta d \vartheta d \varphi=z
$$

i.e. it equals the number $z$ of poles of the considered type in the semi-space (per crystal). For a random orientation distribution, the pole density has for all directions the same value, $D_{r}(P)=z / 2 \pi$.

In a corresponding way, we now define an orientation density ${ }^{22} D(R)$ in the spherical 3-dimensional orientation space (Figure $1 \mathrm{~b}$ ) by

$$
D(R)=\frac{d H(R)}{d \Omega \cdot d \psi}
$$

Here $d H(R)$ is proportional to the volume fraction with a rotation axis in the space angle element $d \Omega$ and the corresponding angle of rotation in the range $d \psi$. The normalization is chosen in such a way that

$$
\int_{\varphi=0}^{2 \pi} \int_{\vartheta=0}^{\pi} \int_{\psi=0}^{\pi \pi} D(R) \cdot \sin \vartheta \cdot d \vartheta \cdot d \varphi \cdot d \psi=24
$$

i.e. it equals the number of rotation axes (per crystal).

In the case of random orientations all spatial directions have equal probability for the direction of the rotation axis, i.e. the orientation density is independent of $\vartheta$ and $\varphi$. According to Deltheil ${ }^{23}$ it depends, however, upon the rotation angle:

$$
D_{r}(R)=\frac{12}{\pi^{2}} \cdot \sin ^{2}\left(\frac{\psi}{2}\right)
$$

Here the normalization factor $12 / \pi^{2}$ has already been chosen so that it complies with Eq. (7). If one inserts Eq. (8) into Eq. (7) and carries out only the first two integrations, one obtains the distribution 
of the rotation angles for a random orientation distribution (compare Part II, Figure 4):

$$
h_{r}(\psi)=\frac{12}{\pi^{2}} \cdot \int D_{r}(R) d \Omega=\frac{24}{\pi} \cdot \sin ^{2}\left(\frac{\psi}{2}\right)
$$

Accordingly, for the density of the rotation axes one obtains for this case by integration over $\psi$ a constant value $h_{r}(v)=12 / \pi$.

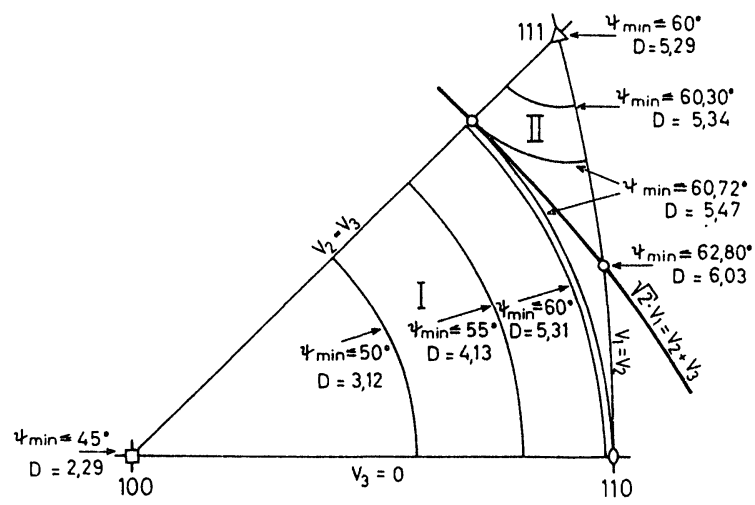

FIGURE 4 Distribution of the limit values of the rotation angles $\psi_{\mathrm{min}}$ and of the density $D\left(\mathbf{v}_{\mathrm{min}}\right)$ of the corresponding rotation axes $\mathbf{v}_{\min }$ for random orientations (compare also Mackenzie ${ }^{16}$ ).

If instead of $\psi$ the coordinate $\psi^{*}$ (see Eq. (1)) is used, one obtains as volume element of the orientation space

$\left(\psi^{*}\right)^{2} \cdot \sin \vartheta d \vartheta d \varphi d \psi^{*}=\frac{12}{\pi^{2}} \cdot \sin ^{2}\left(\frac{\psi}{2}\right) \cdot \sin \vartheta d \vartheta d \varphi d \psi$

i.e. for a random orientation distribution (see Eq. (8)) one obtains a constant orientation density $D_{r}\left(R^{*}\right)=1$. If the stereographic coordinate $\psi^{* *}$ instead of $\psi$ is used, it follows from Eqs. (8) and (1) for the orientation density at random distribution

$$
D_{r}\left(R^{* *}\right)=\frac{192}{\pi^{2}} \cdot \frac{1}{\left[1+\left(\psi^{* *}\right)^{2}\right]^{3}}
$$

For the case of a random orientation distribution the distribution of the rotations with the smallest rotation angle $\left(R_{\min }\right)$ has been calculated by Mackenzie ${ }^{16,17}$ and Handscomb. ${ }^{18}$ By extending the integration (9) over only that part of the orientation space occupied by the $R_{\min }$, one obtains the distribution $h_{r}\left(\psi_{\min }\right)$, which assumes a maximum at $45^{\circ}$ (see also Part II, Figure 6). The corresponding integration over $\psi$ leads to the (then no longer constant) distribution of the rotation axes $\mathbf{v}_{\min }$.
This function $h_{r}\left(\mathbf{v}_{\min }\right)$ is indicated for a unit triangle in Figure 4. This function $h_{r}\left(\mathbf{v}_{\min }\right)$ (as well as the upper limit value $\psi_{\min }$ ) increases perpendicular to concentric circles around $\langle 100\rangle$ - and $\langle 111\rangle$-axes with increasing distance from these poles. It reaches its highest values on the largest circle (thick line) for which $\sqrt{ }(2) v_{1}=v_{2}+v_{3}\left(v_{1}, v_{2}\right.$ and $v_{3}$ are the Cartesian coordinates of the rotation axis unit vector v).

The pole density $D(P)$ can be obtained from the orientation density $D(R)$ as a sum of $2 z$ line integrals :

$$
D(P)=\frac{2 z}{24} \sum_{i=1}^{2 z} \int_{s_{i}} D(R) d s
$$

Let us consider, as an example, a $\{100\}$ pole figure. All rotation axes, which contribute to the pole density at a certain point $Z^{\prime}$ by transforming the standard pole $Z$ into $Z^{\prime}$ are situated on the largest circle (here $G_{3}$ ) which lies symmetrically between $Z^{\prime}$ and $Z$. To each rotation axis $\mathrm{v}$ on this circle belongs another rotation angle $\psi$ (see Eq. (2)), so that this circle corresponds to a path $s_{i}$ in the orientation space. The integration in Eq. (12) must be carried out along this path. Since one has $2 z$ (i.e. here 6) poles of the standard position (the positive and negative directions of the axes must be counted separately) which can be brought into $Z^{\prime}$, one obtains $2 z$ of such circles and, therefore, $2 z$ terms in Eq. (12). $\dagger$ The factor $2 z / 24$ results from the condition that in the case of random orientation the integration over the entire pole sphere (on the left side of Eq. (12)) leads to the value $2 z$ (Eq. (5)) and the integration over the entire orientation space (on the right side of Eq. (12)) yields the value 24 (Eq. (7)). Equation (12) shows again that from the orientation distribution $D(R)$ the pole density distribution $D(P)$ can be unequivocally derived, but not $D(R)$ from $D(P)$.

\section{THE ORIENTATION DISTANCE AND THE DESCRIPTION OF SCATTER AROUND AN IDEAL ORIENTATION}

For the purpose of describing the orientation difference between two crystals by only one parameter the disorientation $\psi_{\min }$ appears to be most

$\dagger$ In the present case of a $\{100\}$ pole figure each orientation corresponding to one of these largest circles is described by 4 different points on this circle (e.g. for the circle transforming $Z$ into $Z^{\prime}$ one has 4 possibilities of transforming the other 2 cube poles into each other). With 6 circles, one arrives again at 24 orientation points per orientation. 
appropriate. According to Eq. (3) it is determined by the 3 angles $\omega_{i}$ between the corresponding cube axes of the two crystals (see Figure 5). As first proposed by Dunn ${ }^{21}$ one often uses only the largest of the 3 angles $\omega_{i}$ which has been defined as "orientation distance $\rho$ " since it is a quantity more simple to determine than the disorientation. (For example, in Figure $5 \rho$ is equal to $\omega_{2}$, the angle between $A_{2}$ and $B_{2}$ ). This definition can easily be applied to other types of axes, e.g. to $\langle 111\rangle$-axes by considering the largest of the 4 angles between corresponding $\langle 111\rangle$ axes as being the orientation distance. $^{24}$ In the following the relationships between $\rho$ and $\psi_{\text {min }}$ will now be derived. The calculations will be limited first to cube axes and to the supposition that the reference crystal is in standard projection.

Equation (2) shows that for a given rotation always that one of the 3 cube axes possesses the largest distance from its original direction, whose angular distance $\lambda$ from the rotation axis $\mathbf{v}$ lies

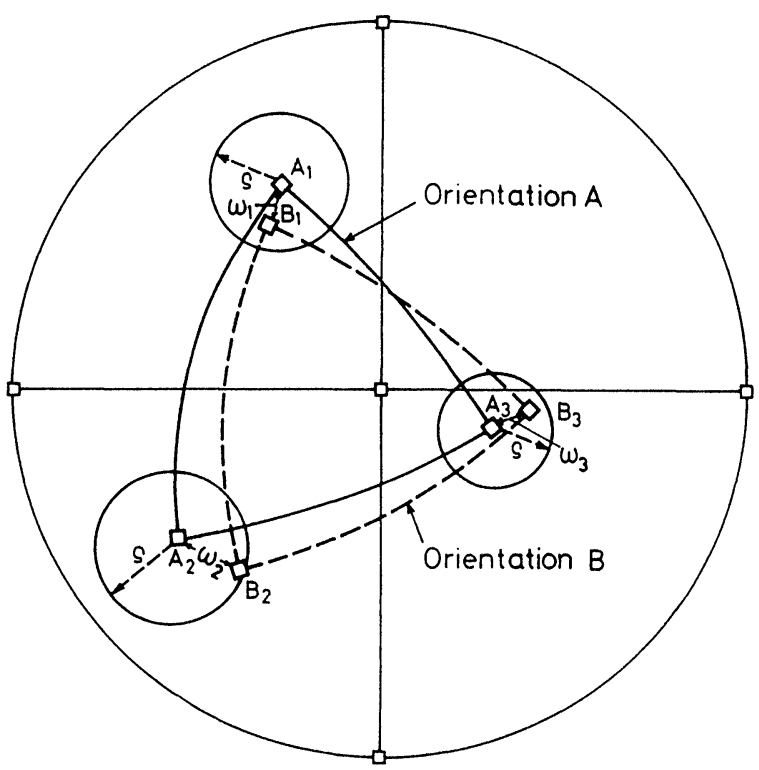

FIGURE 5 Definition of the orientation distance $\rho$ between the orientations $B$ and $A$ ( $\rho$ is the largest of the 3 angles $\omega_{i}$ between the corresponding poles of $A$ and $B$; here $\left.\rho=\omega_{2}\right)$.

closest to $\pi / 2$. Only this cube axis determines the orientation distance $\rho$. (In Figure 6, where the rotation axis characterized by $\varphi$ and $\lambda$ is considered, it is the axis [100]). With $v_{1}$ being the component of the rotation axis vector $\mathbf{v}$ in the direction of this cube axis, and setting $\cos \lambda=v_{1}$ (see Figure 6) and $\omega=\rho$, one obtains from Eq. (2)

$$
\psi_{\min }=2 \arcsin \left(\frac{\sin (\rho / 2)}{\left(1-v_{1}^{2}\right)^{1 / 2}}\right)
$$

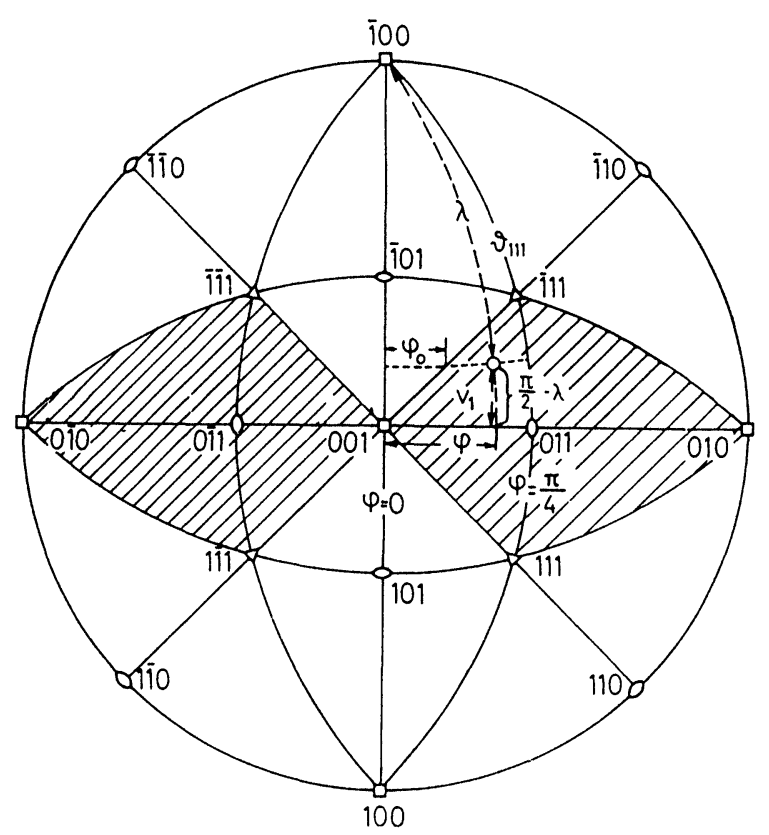

FIGURE 6 Derivation of the relationship between the orientation distance $\rho$ of two crystals, and the rotation coordinates $(\varphi, \vartheta, \psi)$.

Equation(13)is valid for all rotation axeslocated in the 8 shaded triangles, since for those [100] represents the most distant cube axis. For the remaining 16 triangles $v_{1}$, in Eq. (13), must be replaced by $v_{2}$ and $v_{3}$, respectively. According to Eq. (13) $\psi$ has its smallest value on $\langle 100\rangle$-zone circles since here the corresponding $v_{i}=0$ (in the case of Figure 6 one has $v_{1}=0$ along the horizontally running [100]-circle):

$$
\psi_{\langle 100\rangle}=\rho
$$

From here, $\psi$ increases with increasing distance from this circle and reaches its maximum value in the direction of the $\langle 111\rangle$-axis:

$$
\psi_{\langle 111\rangle}=2 \arcsin \left(\frac{\sin (\rho / 2)}{(2 / 3)^{1 / 2}}\right) \approx 1.22 \rho
$$

(Here, the error of approximation made in the second part of this equation is smaller than $1 \%$ for $\rho \leqq 60^{\circ}$ ). 


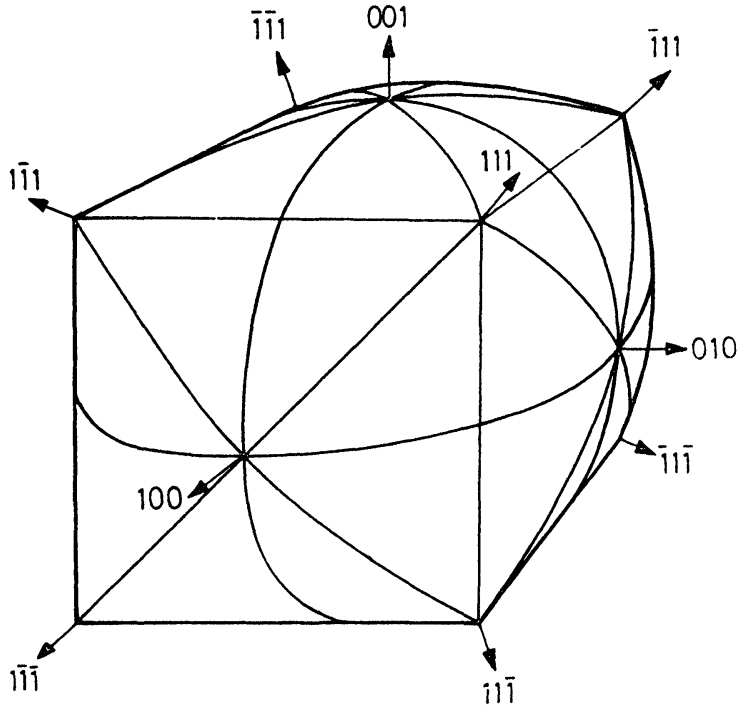

FIGURE 7 The area in the sphere-shaped orientation space $\{\mathbf{v}, \psi\}$ corresponding to a constant orientation distance, $\rho=$ const., with respect to the center orientation.

In the spherical orientation space $\{\mathbf{v}, \psi\}$ Eq. (13) defines for a given $\rho$ a body as shown in Figure 7. It can approximately be described as a sphere of the radius $\psi=\psi_{\langle\mathbf{1 0 0}\rangle}=\rho$, having pyramidal elevations in the $\langle 111\rangle$-directions, or, otherwise, as a cube with an edge length $2 \cdot \psi_{\langle 111\rangle} / \sqrt{ } 3 \approx 1.41 \cdot \rho$ whose surfaces are bowed out to such extent that the circumferential lines parallel to cube faces describe circles with the radius $\psi_{\langle 100\rangle}=\rho$. One recognizes that within a deviation of $\pm 10 \%$ the orientation distance is independent of the position of the rotation axis and roughly given by the size of the rotation angle.

If the orientation space is occupied by orientations with random distribution, the probability, $W(\rho)$, to find an orientation at a distance $\leqq \rho$ from the standard position is equal to the probability of its falling into the interior of the body, Figure 7. This permits an exact calculation of this quantity $W(\rho)$, which had been determined already approximately by Dunn ${ }^{21}$ by considering the individual poles (see also ref. 25). For that purpose this body is transformed into the orientation space $\left\{\mathbf{v}, \psi^{*}\right\}$ with $\psi^{*}$ as length of the radius vector, since then the orientation density for a random distribution is $D_{r}\left(R^{*}\right)=1$ (Eq. (10)) and $W(\rho)$ given directly by the volume of this body. A simple estimate can then be obtained by approximating the body by a sphere of the radius $\psi^{*}$

$$
W(\rho) \approx \frac{4}{3} \pi \cdot\left(\psi^{*}\right)^{3} \approx k \cdot \rho^{3}
$$

Replacing $\psi^{*}$ by Eq. (1) and setting $\psi=\rho$, it leads to $k=1.27$, which corresponds to a too small volume.

The exact calculation has been carried out in Appendix I and gives also, in a very good approximation, the volume proportional to $\rho^{3}$ (maximum deviation $=2.7 \%$ for $\rho=45^{\circ}$ ). It gives further, $k=1.42$ for $\{100\}$-poles and $k=1.38$ for $\{111\}$ poles. Using these $k$-values Eq. (16) defines a radius $\psi^{*}=\psi_{\text {eff }}^{*}$, which leads to a sphere of the same volume as that of the original body. With $k=1.4$ one obtains $\psi_{\text {eff }}=1.03 \rho$, i.e. one finds that here also the orientation distance $\rho$ is about equal to the rotation angle $\psi_{\text {min }}$.

In experimentally determined orientation distributions one often finds orientation scatter around a center orientation ("ideal orientation"). If this scatter has almost spherical symmetryt, i.e. if the distribution depends only upon $\psi$ and not upon $\mathbf{v}$ it is often sufficient to describe it one-dimensionally using the orientation distance with respect to the ideal orientation instead of using the complete 3-dimensional orientation distribution. In such a case one can experimentally determine the (integral) frequency distribution $H(\rho)$, which gives the fraction of crystals having an orientation distance $\leqq \rho$ from the ideal orientation. By comparing the measured distribution $H(\rho)$ to the distribution $W(\rho)$, which is calculated for random orientations (Eq. (16)) a "preference factor"

$$
Q(\rho)=\frac{H(\rho)}{W(\rho)}
$$

can be defined ${ }^{21,25}$ which represents a measure for the degree of accumulation around an ideal orientation.

In general, the ideal orientations do not coincide with the standard orientation as had been assumed in the foregoing. Since $\rho$ always refers to the ideal orientation and $\psi$ to the standard orientation, the simple relationship Eq. (13) is no longer valid in this general case. Moreover, by the transformation of the ideal orientation from the standard position $(\psi=0)$ into an asymmetrical orientation $\left(\psi=\psi_{0}\right)$, the shells around the ideal orientation which characterize the scattering, are being distorted. Therefore, in the case of ideal orientations deviating from the center of the orientation space, certain difficulties arise in evaluating experimental results: (i) the

$\dagger$ This always has to be examined, e.g. Perlwitz, Pitsch, Lücke ${ }^{1}$ found strongly ellipsoidal scattering in rolling textures of copper and brass. 
symmetry of the scattering is no longer simply recognizable, e.g. a spherical scattering around the center looks approximately ellipsoidal if the ideal orientation is situated outside the center; (ii) the angle $\bar{\psi}$ obtained by averaging the rotation angles $\psi$ of all crystals of the accumulation deviates from the angle $\psi_{0}$ for the ideal orientation (if $\psi_{0} \neq 0$ ) even in the case that the scattering would be spherical. These two effects are treated in Appendix II.

If an orientation distribution contains more than one accumulation there is the possibility that the scatter regions overlap. While the extent of overlapping can be directly recognized in a 3-dimensional orientation space, it is less easily recognizable in a pole figure. This problem, which is also important for the evaluation of experiments, is treated in Appendix III.

\section{NUMERICAL DESCRIPTION OF ORIENTATIONS BY ROTATION MATRICES}

For the numerical determination of orientation relationships it is useful to present the orientations of cubic crystals by $3 \times 3$ orthogonal matrices $\mathbf{R}^{25,28}$ with the determinant $\operatorname{Det}(\mathbf{R})=+1$ :

$$
R=\left(\begin{array}{lll}
r_{11} & r_{12} & r_{13} \\
r_{21} & r_{22} & r_{23} \\
r_{31} & r_{32} & r_{33}
\end{array}\right)
$$

Such matrices which are known as rotation matrices, can be used to describe the above treated rotation of the reference system (corresponding to the unit matrix $\mathbf{U}$ ) into the coordinate system of the crystal axes. $\dagger$

The three columns of the matrix give the Cartesian coordinates of the unit vectors $\mathbf{r}_{i}$ of the cube axes

$$
\mathbf{R}=\left(\mathbf{r}_{1}, \mathbf{r}_{2}, \mathbf{r}_{3}\right)
$$

with $\quad \mathbf{r}_{1}=\mathbf{u}_{1} \cdot r_{11}+\mathbf{u}_{2} \cdot r_{21}+\mathbf{u}_{3} \cdot r_{31}$ etc.

with $\mathbf{u}_{1}, \mathbf{u}_{2}, \mathbf{u}_{3}$ as the unit vectors of the axes of the reference system. If the rotation $\mathbf{R}$ occurs relative to an orientation which corresponds to a rotation $\mathbf{A}$ relative to the reference system, one obtains a rotation

$$
\mathbf{B}=\mathbf{A} \cdot \mathbf{R}
$$

$\dagger$ Also in this representation, only three independent parameters occur, since between the 9 matrix elements $r_{i k}$ one has 3 equations of normalization and 3 equations of orthogonality. relative to the reference system (for $\mathbf{A}=\mathbf{U}$ one gets again $\mathbf{B}=\mathbf{R})$. Hence the orientation of $B$ with respect to $A \ddagger$ :

$$
\mathbf{R}=\mathbf{A}^{-1} \cdot \mathbf{B}=\mathbf{A}^{\prime} \cdot \mathbf{B}
$$

Axis and angle of rotation can be obtained from the matrix elements $r_{i k}$ as follows. Since at the rotation $R$ the rotation axis $\mathrm{v}$ is retained one has

$$
\mathbf{R} \cdot \mathbf{v}=\mathbf{v}
$$

i.e. $\mathbf{v}$ is a eigenvector of the matrix $\mathbf{R}$. The same applies for the reversed rotation, hence

and

$$
\mathbf{R}^{-1} \cdot \mathbf{v}=\mathbf{R}^{\prime} \cdot \mathbf{v}=\mathbf{v}
$$

$$
\left(\mathbf{R}-\mathbf{R}^{\prime}\right) \cdot \mathbf{v}=\mathbf{R}^{*} \cdot \mathbf{v}=0
$$

with $r_{i k}=r_{i k}-r_{k i}$. Equation (23) represents a system of three homogeneous linear equations for the components $v_{1}, v_{2}, v_{3}$ of $\mathbf{v}$ with the determinant Det $\left(\mathbf{R}^{*}\right)=0$. This means, this system corresponds to two independent equations so that together with the normalization condition $v_{1}^{2}+v_{2}^{2}+v_{3}^{2}=1$ one has 3 equations from which the components $v_{\mathrm{i}}$ can be calculated individually. One finds

$$
\begin{gathered}
v_{1}=K \cdot\left(r_{23}-r_{32}\right) ; \quad v_{2}=K \cdot\left(r_{31}-r_{13}\right) ; \\
v_{3}=K \cdot\left(r_{12}-r_{21}\right)
\end{gathered}
$$

with

$$
\frac{1}{K}=\left[\left(r_{23}-r_{32}\right)^{2}+\left(r_{31}-r_{13}\right)^{2}+\left(r_{12}-r_{21}\right)^{2}\right]^{1 / 2}
$$

The rotation angle $\psi$ can be obtained from the (inversant) trace of the matrix

$$
\sum_{j} r_{j j}=1+2 \cos \psi
$$

in agreement with Eq. (3).

If one has found one of the possible 24 rotations, e.g. $\mathbf{R}_{1}$, the others can be obtained by interchanging-prior to application of this rotation $\mathbf{R}_{\mathbf{1}}$-the cube axes of the reference system. This can be achieved by applying suitable rotations $\mathbf{T}_{i}$ which

$\ddagger$ For the orthogonal and normalized matrices which are used here, the inverse matrix $A^{-1}$, which describes the reversed rotation, is equal to the transposed matrix $A^{\prime}$, which is defined by $a_{i k}^{\prime}=a_{k i}$. Thus we have here

$$
\mathbf{A} \cdot \mathbf{A}^{\prime}=\mathbf{U}=\left(\begin{array}{l}
100 \\
010 \\
001
\end{array}\right)
$$


are given by the 24 cubic symmetry operations. $\dagger$ The 24 identical orientations are thus arrived at by a sequence of two rotations

$$
\mathbf{R}_{i}=\mathbf{R}_{1} \cdot \mathbf{T}_{i} \quad \text { with } \quad i=1,2, \cdots, 24
$$

The corresponding coordinates $(\mathbf{v}, \psi)_{i}$ are obtained from $\mathbf{R}_{i}$ again by Eqs. (24) to (26). The orientation distance $\rho$ is given by the smallest of the diagonal elements of the matrix $\mathbf{R}_{\text {min }}$

$$
\cos \rho=\operatorname{Min}\left(r_{\min }\right)_{j j}
$$

In Part II it will be shown, with the aid of an example, how to determine numerically such rotation matrices and the rotational coordinates from experimental data and how to apply these to a rotational description of orientation distributions.

\section{Appendix I: DISTRIBUTION OF THE ORIENTATION DISTANCES AT RANDOM ORIENTATION DISTRIBUTION}

According to Eqs. (13) and (1) modified rotation angle $\psi_{\min }$ is given as a function of the orientation distance $\rho$ by

$$
\begin{aligned}
\psi^{*}=\left\{\frac { 1 8 } { \pi ^ { 2 } } \cdot \left[2 \arcsin \left\langle\frac{\sin (\rho / 2)}{\sin \lambda}\right\rangle\right.\right. \\
\left.\left.-\sin \left(2 \arcsin \left\langle\frac{\sin (\rho / 2)}{\sin \lambda}\right\rangle\right)\right]\right\}^{1 / 3}
\end{aligned}
$$

Since in the case of random orientation distribution one has $D_{r}\left(R^{*}\right)=1$ in the $\left(\mathbf{v}, \psi^{*}\right)$-space, the probability $W\left(\rho_{m}\right)$ to find orientations with $\rho \leqq \rho_{m}$ is

$$
W\left(\psi_{m}^{*}\right)=\int_{\varphi=0}^{2 \pi} \int_{\vartheta=0}^{\pi} \int_{\psi^{*}=0}^{\psi m^{*}}\left(\psi^{*}\right)^{2} \cdot \sin \vartheta d \vartheta d \varphi
$$

$\uparrow$ Since these operations can be reduced to the three basic rotations

$\mathrm{T}_{100}=90^{\circ}[100] ; \quad \mathrm{T}_{111}=120^{\circ}[111] ; \quad \mathrm{T}_{110}=180^{\circ}[110]$ one has generally

$$
\mathbf{T}_{i}=\mathbf{T}_{100}^{r} \cdot \mathbf{T}_{111}^{s} \cdot \mathbf{T}_{110}^{t}
$$

with $i=1,2, \ldots, 24 ; r=0,1,2,3 ; s=0,1,2 ; t=0,1$. In particular, one has for $r=s=t=\sigma \mathrm{T}_{1}=\mathrm{U}$, and for the fundamental interchange matrices

$\mathbf{T}_{100}=\left(\begin{array}{lll}1 & 0 & 0 \\ 0 & 0 & \overline{1} \\ 0 & 1 & 0\end{array}\right) \mathbf{T}_{111}=\left(\begin{array}{lll}0 & 0 & 1 \\ 1 & 0 & 0 \\ 0 & 1 & 0\end{array}\right) \mathbf{T}_{110}=\left(\begin{array}{lll}0 & 1 & 0 \\ 1 & 0 & 0 \\ 0 & 0 & \overline{1}\end{array}\right)$ with $\psi_{\min }^{*}$ being the value resulting from Eq. (29) for $\rho=\rho_{m}$.

Because of the crystallographic symmetry, one can limit the integration to one of the 48 unit triangles, for instance, to triangle [001]-[011]-[111] of Figure 6. Setting $\lambda=\vartheta$ and carrying out the integration over $\psi^{*}$ and $\varphi$, the latter from $\varphi_{0}$ to $\pi / 4$ :

$$
W\left(\psi_{m}^{*}\right)=48 \int_{\vartheta_{111}}^{\pi / 2}\left(\frac{\pi}{4}-\varphi_{0}\right) \cdot \frac{1}{3}\left(\psi_{m}^{*}\right)^{3} \cdot \sin \vartheta d \vartheta
$$

By inserting for the boundaries of the triangle $\sin \varphi_{0}=\operatorname{ctg} \vartheta$ and $\operatorname{ctg} \vartheta_{111}=\sqrt{ }(2) / 2$ and applying the substitution $\operatorname{ctg} \vartheta=\sin x$, one finally obtains

$$
\begin{aligned}
W\left(\rho_{m}\right)= & \frac{288}{\pi^{2}} \cdot \int_{x=0}^{\pi / 4} \frac{\left(\frac{\pi}{4}-x\right) \cdot \cos x}{\left(1+\sin ^{2} x\right)^{3 / 2}} \\
& \times\left[F\left(x, \rho_{m}\right)-\sin \left\{F\left(x, \rho_{m}\right)\right\}\right] d x
\end{aligned}
$$

with

$$
F\left(x, \rho_{m}\right)=2 \arcsin \left[\left(1+\sin ^{2} x\right)^{1 / 2} \cdot \sin \left(\rho_{m} / 2\right)\right]
$$

This integral cannot be solved analytically. However, for small $\rho$ both terms in the integral can be expanded so that with $x-\sin x=x^{3} / 6$ the easily solvable integral

$$
W\left(\rho_{m}\right)=\frac{288}{\pi^{2}} \cdot \frac{\rho_{m}^{3}}{6} \cdot \int_{x=0}^{\pi / 4}\left(\frac{\pi}{4}-x\right) \cos x d x \approx 1.424 \cdot \rho_{m}^{3}
$$

is obtained in agreement with Eq. (16). A numerical evaluation of integral (32) reveals that the approximation (33) is very good; even for $\rho_{m}=45^{\circ}$ it leads to a value only $2.7 \%$ above the exact one. (For $\rho_{m}>45^{\circ}$ Eq. (32) becomes invalid, as then two $\{100\}$-poles of the same orientation may fall into the same circle of scattering, a possibilty not been considered in the derivation of Eq. (30)).

A similar calculation can be carried out, if one defines $\rho$ as the largest of the four angles between the corresponding $\langle 111\rangle$-axes. Also, in this case Eq. (29) is valid; one only has to insert for $\lambda$ the angle closest to $\pi / 2$ of the four angles between the rotation axes and the four $\langle 111\rangle$-axes. Also here, the integration can be carried out over a unit triangle and results again in an expression of the type of Eq. (16) with $k=1.382$. This expression is valid up to $\rho_{m}=35.5^{\circ}$ (half the distance of two $\{111\}$-poles). 
Appendix II: SCATTER AROUND AN IDEAL ORIENTATION WHICH DOES NOT COINCIDE WITH THE STANDARD ORIENTATION

As a simple example of an orientation distribution in the spherical orientation space (Fig. 1b) we consider a sphere of the radius $\psi_{s}$ around the center of this space. It may contain $N$ orientations leading to an orientation density $p D_{r}(\psi)$ (see Eq. (8)) inside the sphere, whereas the rest of the orientation space is not occupied. $\dagger$ After a rotation of the
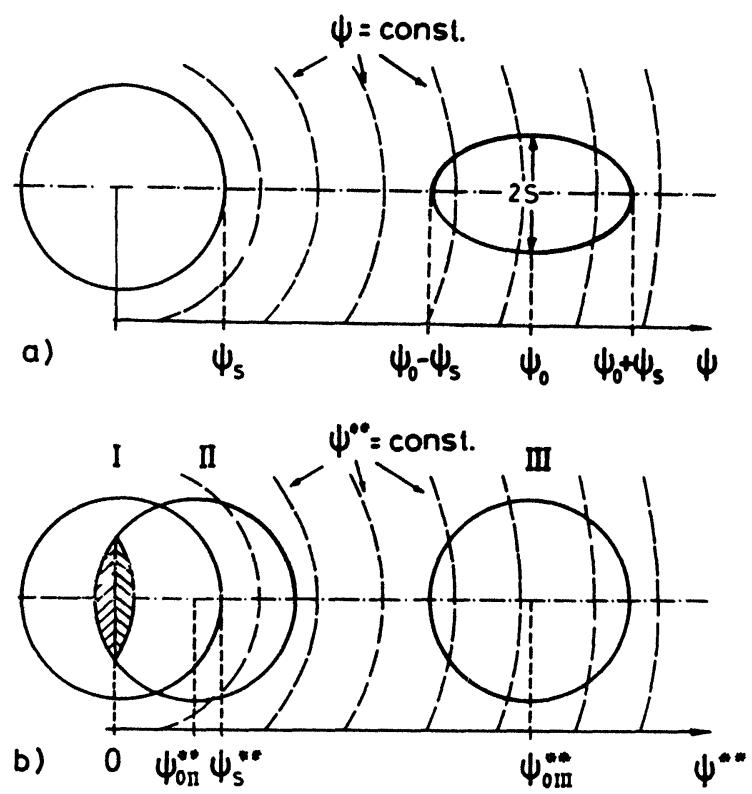

FIGURE 8 Transformation of a sphere with the radius $\psi_{s}$ around the center of the spherical orientation space (sphere I) by a rotation of the angle $\psi_{0}>\psi_{s}$ (schematic) (a) in the $\{\mathbf{v}, \psi\}$ space, (b) in the $\left\{\mathbf{v}, \psi^{* *}\right\}$ space. Here also a sphere corresponding to an angle $\psi_{0}<\psi_{s}$ is plotted (II).

coordinate system around the angle $\psi_{0}$ around an axis $\mathbf{v}_{0}$ the ideal orientation originally situated in the center will be located at a point in the orientation space characterized by $v_{0}$ and $\psi_{0}$ and again be surrounded by a scattering zone.

This zone, however, is no longer spherically shaped. In radial direction the diameter $2 \psi_{s}$ has been maintained since in the direction of the rotation axis the rotation angles can simply be added. In azimuthal direction, however, the scattering zone

$\dagger$ According to Eq. (31) the normalization factor $p$ follows from $p W\left(\psi_{s}^{*}\right)=1\left(\right.$ with $\left.\psi_{m}=\psi_{s}\right)$. is smaller, since the orientation density increases with increasing $\psi$ (Eq. (8)) and, therefore, the volume required for $N$ orientations decreases. For small $\psi_{s}$ and not too small $\psi_{0}$ one can approximate the scattering body by a rotational ellipsoid (Figure 8a) with the length of the large axis being $2 \psi_{s}$ and that of two small axes being $2 s$, with $s$ defined by

$$
\pi \cdot s^{2}=\frac{3}{\pi} \cdot \psi_{s}^{2} / D_{r}\left(\psi_{0}\right)
$$

This relationship is obtained by equating the number of orientations in the ellipsoid to that in the sphere around zero (see Eqs. (16) and (1)), assuming that the density in the whole ellipsoid is approximately given by $D_{r}\left(\psi_{0}\right)$, i.e. by that at the point $\left(\mathbf{v}_{0}, \psi_{0}\right)$,

$\frac{4 \pi}{3} \cdot \psi_{s} \cdot s^{2} \cdot p \cdot D_{r}\left(\psi_{0}\right)=\frac{4 \pi}{3} \cdot p \cdot\left(\psi_{s}^{*}\right)^{3} \approx \frac{4}{\pi} \cdot p \cdot \psi_{s}^{3}$

In this approximation, in which $\psi_{0}=\bar{\psi}$ represents the center of the ellipsoid, also the distribution of the $\psi$-values can be easily derived. With $\Delta \psi=$ $\left|\psi-\psi_{0}\right|$ and with Eqs. (34) and (35) one obtains the differential frequency (normalized to the total number $N$ )

$h(\Delta \psi)=2 p \cdot D_{r}\left(\psi_{0}\right) \cdot \pi \cdot r^{2}=\frac{3}{2} \cdot \frac{1}{\psi_{s}} \cdot\left\{1-\left(\frac{\Delta \psi}{\psi_{s}}\right)\right\}$

Here, $r$, given by

$$
\left(\frac{\Delta \psi}{\psi_{s}}\right)^{2}+\left(\frac{r}{s}\right)^{2}=1
$$

is the radius of the (approximately plane) circular sections of the ellipsoid at constant $\psi$. From Eq. (36) one obtains the integral frequency

$$
\begin{aligned}
H(\Delta \psi) & =\int_{0}^{\Delta \psi} h(\Delta \psi) d \psi \\
& =\frac{3}{2} \cdot \frac{\Delta \psi}{\psi_{s}} \cdot\left\{1-\frac{1}{3}\left(\frac{\Delta \psi}{\psi_{s}}\right)^{2}\right\}
\end{aligned}
$$

and in particular, for the slope of $H(\Delta \psi)$ at small $\Delta \psi$

$$
h_{0}=\frac{3}{2 \cdot \psi_{s}}
$$

For more exact calculations it is more practical to use the orientation space with the radius vector $\psi^{* *}$ (Eq. (1)) instead of $\psi$, since then-as can be shown ${ }^{20}$ - the transformation turns spheres into 
spheres (only their radius increases with increasing $\left.\psi_{0}\right)$. Moreover, one has to consider that the density $p D_{r}\left(\psi^{* *}\right)$ is not constant within the sphere (Eq. (8)) and that the volume elements for deriving the expression for $h\left(\psi^{* *}\right)$ must be taken along the surface of spheres around the origin characterized by $\psi^{* *}=$ const. (see Fig. 8 b). One finds then the equation

$$
\begin{aligned}
& \frac{2}{W\left(\psi_{s}^{* *}\right)} \cdot \frac{d H\left(\Delta \psi^{* *}\right)}{d \psi^{* *}} \\
& =\frac{192}{\psi_{s}^{3}} \cdot \frac{\left(\psi^{* *}\right)^{2}}{\left[1+\left(\psi^{* *}\right)^{2}\right]^{3}} \cdot\left\{1-\frac{\cos \left(\frac{\psi_{s}}{2}\right)+\cos \left(\frac{\psi_{0}}{2}\right)}{2\left(\psi^{* *}\right) \sin \left(\frac{\psi_{0}}{2}\right)}\right. \\
& \left.\times\left[\left(\psi^{* *}\right)^{2}+\frac{\cos \left(\frac{\psi_{s}}{2}\right)-\cos \left(\frac{\psi_{0}}{2}\right)}{\cos \left(\frac{\psi_{s}}{2}\right)+\cos \left(\frac{\psi_{0}}{2}\right)}\right]\right\} \quad(40)
\end{aligned}
$$

where $\psi^{* *}$ must be replaced by $\psi_{0}$ and $\Delta \psi$. For small values of $\psi_{s}$ Eq. (39) is retained.

Such exact calculations lead also to the result that, in contrast to the approximative treatment, the average angle of rotation $\bar{\psi}$ does no longer coincide with the ideal value $\psi_{0} . \bar{\psi}$ is defined by

$\bar{\psi}=4 \overline{\operatorname{arctg}}\left(\psi^{* *}\right)=\frac{\oint D_{r}\left(\psi^{* *}\right) \cdot \operatorname{arctg}\left(\psi^{* *}\right) d V}{\oint D_{r}\left(\psi^{* *}\right) d V}$

with the integration taking place over the volume of the sphere in the $\left\{\mathbf{v}, \psi^{* *}\right\}$-space. The evaluation of this integral will lead to the difference $\delta=\bar{\psi}-\psi_{0}$ as a function of $\psi_{s}$ and $\psi_{0}$. Since, however, we are dealing only with a model distribution, the exact evaluation will not be carried out, and only a qualitative discussion of the principal features will be given.

We consider $\psi_{s}=$ const. and vary $\psi_{0}$ (see Figure $8 \mathrm{~b}$ ). For $\psi_{0}=0$ is also $\delta=0$, since at the integration the regions of the sphere with positive and negative $\psi^{* *}$ just cancel each other (sphere I). With $0<\psi_{0}<\psi_{s}$ one has $\delta>0$, since the regions cancelling each other (shaded area) become smaller (sphere II). Finally, at $\psi_{0}=\psi_{s}$, the surface of the sphere reaches the zero point, so that for $\psi_{0}>\psi_{s}$ all cancelling is abolished and no further increase of $\delta$ possible (sphere III). On the other hand, with increasing $\psi_{0}$ the curvature of the lines $\psi^{* *}=$ const (see sphere III) as well as the density $D_{r}\left(R^{* *}\right)$
(Eq. (11)) decreases causing also a decrease of $\delta$. The superposition of the increasing and the decreasing influence leads to a curve $\delta\left(\psi^{* *}\right)$ having a maximum approximately at $\psi_{0}=\psi_{s}$ as indicated in Figure 9. A numerical calculation carried out for $\psi_{s}=23^{\circ}$ yielded for $\psi_{0}=\psi_{s}$ (i.e. approximately for the peak) $\delta=4.5^{\circ}$ and for $\psi_{0}=120^{\circ}$ only

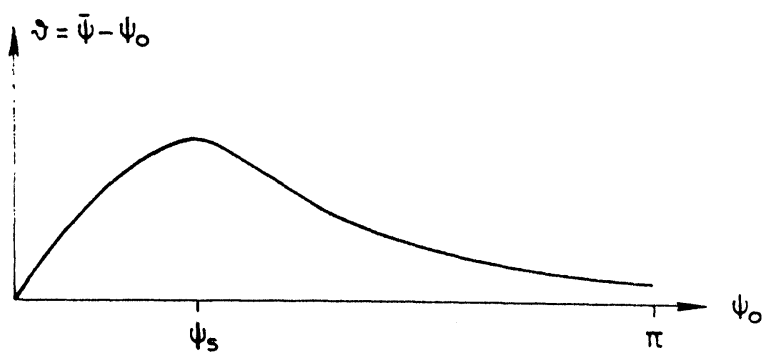

FIGURE 9 Deviation $\delta$ of the average value $\bar{\psi}$ of the rotation angles from the ideal angle $\psi_{0}$ as a function of $\psi_{0}$ for spherical orientation scattering with the scatter radius $\psi_{s}$ (schematic).

$\delta=0.48^{\circ}$. The magnitudes of these values should be maintained even if instead of this model distribution a more real orientation distribution is used in which $\psi_{s}$ possesses the character of a half value radius.

\section{Appendix III: OVERLAPPING OF SCATTER ZONES}

Since 2 orientations can coincide only if all three cube poles coincide, one obtains a true overlapping of the scatter regions of two orientation accumulations only if the scatter regions of all 3 cube poles overlap. If the scatter region of only one pole has no overlapping with any scatter region of the poles of the other accumulation, the two accumulations cannot contain a common orientation.

In order to elucidate the conditions the spherical orientation space (Figure 1b) is considered. Let one scatter sphere (radius $\psi_{s}$ ) be around the center and a second scatter body be obtained by rotating the first one around an angle $\psi<\psi_{s}$ so that overlapping is obtained (for instance sphere $I$ and sphere II in Figure 8). If the line connecting the ideal orientations I and II which indicates the direction of the rotation axis, happens to be a cube axis, one obtains a complete overlapping of the scatter region of the cube poles corresponding to this axis. The degree of overlapping is then determined solely by the overlapping of the scatter zones 
of the two other $\{100\}$-poles, and is given by the rotation angle $\psi$, i.e. by the length of the vector connecting the two ideal orientations.

If, however, the rotation axis does not coincide with a cube axis one has no longer a complete overlapping of the scattering zones of these poles. Since during the rotation leading from I to II the cube pole closest to the rotation axes undergoes the smallest, and the one closest to $90^{\circ}$ to it the largest change in direction, the overlapping of the scatter regions of the first pole is largest and that of the second smallest. In the case where the angle between rotation axes and the next located cubic axes is not too large, the overlapping of the orientation scattering is still determined by the overlapping of pole scatterings of the $\{100\}$-poles located nearest to $90^{\circ}$ to the rotation axis. In this case, i.e. if the overlapping of the 3 pole regions is very different, the smallest one of the 3 overlappings represents a measure for the overlapping in the orientation space.

The consequences for the evaluation of the experimental results arising from such overlappings will be considered in Part II.

\section{REFERENCES}

1. K. Lücke, H. Perlwitz, and W. Pitsch, Phys. Stat. Sol. 7, 733 (1964)

2. R. O. Williams, Trans. Met. Soc. AIME 242, 105 (1968).

3. A. S. Viglin, Fiz. twerdoga tela 2, 2463 (1960).
4. H.-J. Bunge, Z. Metallkde. 56, 872 (1965).

5. H.-J. Bunge, Phys. Stat. Sol. 26, 167 (1968).

6. H.-J. Bunge, Kristall u. Techn. 3, 439 (1968)

7. H.-J. Bunge and W. T. Roberts, J. Appl. Cryst. 2, 116 (1969).

8. H.-J. Bunge and T. Leffers, Scripta Met. 5, 143 (1971).

9. R. J. Roe, J. Appl. Phys. 36, 2024 (1965).

10. R. J. Roe, J. Appl. Phys. 37, 2069 (1966).

11. P. R. Morris and A. J. Heckler, Adv. in X-Ray Analysis 11, 454 (1968).

12. J. S. Kallend and G. J. Davies, J. Inst. Met. 97, 350 (1969).

13. A. J. Heckler and W. G. Granzow, Met. Trans. 1, 2089 (1970).

14. G. J. Davies, D. J. Goodwill, and J. S. Kallend, J. Appl. Cyst.4,67 (1971).

15. J. K. Mackenzie and M. J. Thomson, J. Appl. Phys. 39, 5503 (1968).

16. J. K. Mackenzie, Biometrika 45, 229 (1958).

17. J. K. Mackenzie, Acta Met. 12, 223 (1964).

18. D. C. Handscomb, Canad.J. Math. 10, 85 (1958).

19. G. Ibe and K. Lücke, to be published.

20. G. Gorn, G. Ibe and K. Lücke, to be published.

21. C. G. Dunn, Phys. Rev. 66, 215 (1944).

22. C. G. Dunn and J. L. Walter, J. Appl. Phys. 31827 (1960).

23. R. Deltheil, 'Probabilités géométriques', (GauthierVillars, Paris, 1926).

24. W. Dietz, A. C. Fraker, G. Ibe and K. Lücke, $Z$. Metallkde. 61, 498 (1970).

25. G. Ibe, Dissertation T. H. Aachen (1965).

26. $\mathrm{K}$. Lücke, in VIIe Colloque de Métallurgie, Paris, (Commissariat à l'Energie Atomique, 1963).

27. G. Ibe and K. Lücke, in Recrystallization, Grain Growth and Textures, (American Society for Metals, 1966) p. 434.

28. F. Lihl and W. Pexa, Z. Angew. Phys. 22, 549 (1967). 